\title{
Identification of ANXA1 as a diagnostic and lymphatic metastasis factor in colorectal cancer
}

\author{
Qing-Hua Liu" ${ }^{1 *}$, Mei Ding ${ }^{2,3 *}$, Jin-Min Sun ${ }^{1}$ and Zu-An Zhu ${ }^{2}$ \\ ${ }^{1}$ Department of Pathology, Xuzhou Medical University, 221004, China \\ ${ }^{2}$ Department of Gastroenterology, Affiliated Hospital of Xuzhou Medical University, 221000, China \\ ${ }^{3}$ Department of Critical-care Medicine, Zoucheng People's Hospital, 273500, China
}

\begin{abstract}
Objective: To determine whether ANXA1 can be served as biomarker for diagnosis in colorectal cancer (CRC) and analyse the correlation of ANXA1 and clinicopathological parameters of CRC patients.

Methods: Tissue array (n=95) CRC specimens were utilised in this study. ANXA1 in cancer and non-cancerous tissues were detected immunohistochemically. The correlations between ANXA1 and clinicopathological parameters of CRC were evaluated. Concentrations of ANXA1 in peripheral blood were detected by enzymelinked immunosorbent assay (ELISA).

Results: ANXA1 was upregulated in CRC tissues than that in non-cancerous tissues $(P<0.05)$. The mean value for ANXA1 level in peripheral blood of CRC patients was significantly higher than that of healthy individuals $(P<0.05)$. Increased ANXA1 was observed in deeper tumor invasion, lymphatic metastasis and late TNM stage of CRC patients.

Conclusions: ANXA1 increased in CRC tissues and in peripheral blood of CRC patients. In addition, increased ANXA1 was correlated with deeper tumor invasion, lymph node metastasis and late TNM stage of CRC patients. Our findings would provide new insights into significant of ANXA1 in diagnosis and lymphatic metastasis of CRC. The underlying mechanism of ANXA1 in CRC progress will be further explored.
\end{abstract}

\section{Introduction}

CRC is the third most common cancer in male and the second in female worldwide. In addition, CRC is the fourth leading cause of cancer mortality worldwide [1]. The treatment of CRC patients with metastases through combination chemotherapy helps improving the prognosis, but overall prognosis for CRC patients remains poor and the 5-year survival rate is less than $19 \%$ in stage IV patients with distant metastases [2]. Therefore, studying the molecular mechanism of CRC cell growth, metastasis and finding key markers is very important for early diagnosis, prognosis and may give insight into clinic targeted therapy of CRC patients.

Annexin a1 (ANXA1), the first characterized member of annexin superfamily contributes to variety cellular biological activities: antiinflammatory effects, cell differentiation and most importantly involved in the process of cancer [3]. ANXA1 has been recognized down regulated in head neck cancer and esophageal cancer, up regulated in pancreatic cancer and mammary adenocarcinoma [4,5]. Researchers are hampered by conflicting roles of ANXA1 in cancers and by lack of demonstrating the specific expression of it.

We therefore systematically investigate the expression of ANXA1 in human CRC solid cancers and matched non-cancerous tissues. Immunohistochemistry were established to detect the tissue-specific expression of ANXA1. Furthermore, we detected the concentrations of ANXA1 in peripheral blood of CRC patients and healthy individuals by ELISA. Our data may provide useful information for ANXA1 used as a diagnostic marker and/or further used as therapeutic targets for CRC patients.

\section{Materials and methods}

\section{Patients and samples}

CRC tissues and non-cancerous tissues (control) were obtained from 95 CRC patients undergoing resection in the Affiliated Hospital, Xuzhou Medical University, China, between 2007 and 2011. These CRC patients were comprised of 54 males and 41 females, aged from 34 to 69 years old (Table 1). No chemotherapy was used in these patients prior to resection. This study was approved by the Human Ethical Committee, the Institutional Review Boards of Affiliated Hospital of Xuzhou Medical University, and written informed consents were obtained.

Between 2016 and 2017, 20 patients with CRC and 20 healthy individuals were included in this study. The peripheral blood samples from CRC patients were collected before resection of primary tumour. This study was approved by the Human Ethical Committee, the Institutional Review Boards of Affiliated Hospital of Xuzhou Medical University, and written informed consents were obtained.

${ }^{*}$ Correspondence to: $\mathrm{Zu}$-An $\mathrm{Zhu}$, Department of Gastroenterology, Affiliated Hospital of Xuzhou Medical University, 84 Huai-Hai Western Road, Xuzhou, 221000, Jiangsu Province, China, Tel: +86 15852162813; E-mail: zhuzuanliu@126.com

Key words: ANXA1, immunohistochemistry, ELISA, lymphatic metastasis

Received: November 12, 2018; Accepted: November 22, 2018; Published: November 26, 2018 
Table 1. Expression of ANXA1 in CRC tissues and in adjacent non-cancerous tissues

\begin{tabular}{|c|c|c|c|c|c|}
\hline Groups & $\mathbf{n}$ & $\begin{array}{c}\text { Positive rate } \\
\text { (\%) }\end{array}$ & $\begin{array}{c}\text { Negative } \\
\text { rate (\%) }\end{array}$ & $\boldsymbol{\chi}^{\mathbf{2}}$ & $\boldsymbol{P}$ \\
\hline CRC & 95 & $71(74.74)$ & $24(25.26)$ & 11.896 & 0.001 \\
\hline Non-cancer & 95 & $48(50.53)$ & $47(49.47)$ & & \\
\hline
\end{tabular}

\section{Immunohistochemistry}

Tissue microarrays had been used the paraffin-embedded specimens of 95 CRC tissues and non-cancerous tissues by a Beecher Instrument (Silver Spring, MD, USA). The sections $(5 \mu \mathrm{m})$ from the tissue microarray blocks were labelled with anti-ANXA1 polyclonal antibody (TA500967, OriGene Technologies, Inc., USA) at dilution of 1:200. HRP- conjugated secondary antibody (12127A07, Beijing Sequoia Jinqiao Biological Technology Co., Ltd.) was used according to the manufacturer's instructions. The specific target(s) was visualized with 3, 3'-diaminobenzidine (DAB) detection kit (Beijing Sequoia Jinqiao Biological Technology Co., Ltd.) and counterstained with hematoxylin. All the slides were examined and scored independently by two pathologists. ANXA1 was stained brown in cytoplasm with or without in nucleus of CRC.

\section{ELISA}

In our study, we prospectively enrolled $20 \mathrm{CRC}$ patients in the Affiliated Hospital, Xuzhou Medical University, China, in 2016-2017. Patients who had received preoperative chemotherapy were excluded. And 20 healthy persons from non-cancer patients were collected as controls in our study. $5 \mathrm{ml}$ peripheral blood was collected from subjects and centrifuged at $3000 \mathrm{rpm}$ for $15 \mathrm{~min}$ at $4^{\circ} \mathrm{C}$, and then the supernatants were stored at $-80^{\circ} \mathrm{C}$ until analysis. According to the manufacturers's instructions of Human ANXA1 (SEE787Hu, CloudClone Corp, Wuhan, China), we determined concentrations of ANXA1 by ELISA kit (Bio-Rad 550, US) at 450nm. All samples were checked in duplicates.

\section{Statistical analysis}

SPSS16.0 statistical software package was used for our statistical analysis. Association between the expression of ANXA1 and the clinicopathological characteristics had been analyzed by using Chisquare test. Student's t test was used for comparing variable between groups in ELISA assay. $P<0.05$ was considered as statistically significant.

\section{Results}

\section{Expression of ANXA1 in CRC tissue}

ANXA1 in CRC tissues was stained in brown mainly located in cytoplasm with or without nuclear staining in immunohistochemistry assay (Figure 1). Additionally, the expression of ANXA1 was significantly increased in CRC compared to non-cancerous tissues $(P<0.05)$ (Table 2 and Figure 2A).

\section{Correlation between ANXA1 in CRC and clinicopathological parameters}

Associations between clinicopathological parameters and ANXA1 are listed in Table 1 and Figures 2B-2D. There was significantly higher expression of ANXA1 in the group of patients with lymph node metastasis, deeper tumour invasion (invasion depth) and late TNM stage. Moreover, no significant differences were observed in ANXA1 with age, gender, differentiation and diameter of CRC.

\section{ANXA1 in peripheral blood of CRC patients and in healthy individuals}

The mean value for ANXA1 in peripheral blood of CRC patients was $5.184 \pm 1.070 \mathrm{ng} / \mathrm{ml}$, which was significantly higher than that of healthy individuals $(1.031 \pm 0.600 \mathrm{ng} / \mathrm{ml})(P<0.05)$ (Figure 3$)$.

\section{Discussion}

Although ANXA1 was an attracting protein in cancer research, the contradictory expression levels in solid tumors and different roles limited its usage as biomarker in diagnosis and therapeutic of cancer in clinic [6-8]. Furthermore, previous studies had reported that ANXA1 was also altered in the same type tumors, e.g., large number reports of contradictory results of ANXA1 in breast cancers [9-11]. In our present study, the expression of ANXA1 in human CRC tissues was explored

Table 2. Comparision of clinicopathological features in CRC patents $(\mathrm{n}=95)$

\begin{tabular}{|c|c|c|c|c|}
\hline \multirow{2}{*}{ Variables } & \multicolumn{4}{|c|}{ ANXA1 staining } \\
\hline & Positive (\%) & Negative (\%) & Total & $P$ \\
\hline \multicolumn{5}{|l|}{ Age } \\
\hline$>55$ & $35(72.92)$ & $13(27.08)$ & 48 & 0.68 \\
\hline$\leq 55$ & $36(76.60)$ & $11(23.40)$ & 47 & \\
\hline \multicolumn{5}{|l|}{ Gender } \\
\hline Male & $43(79.63)$ & $11(20.37)$ & 54 & 0.208 \\
\hline Female & $28(68.29)$ & $13(31.71)$ & 41 & \\
\hline \multicolumn{5}{|c|}{ Tumor differentiation } \\
\hline Well & $14(70.00)$ & $6(30.00)$ & 20 & 0.86 \\
\hline Moderate & $48(76.19)$ & $15(23.81)$ & 63 & \\
\hline Poor & $9(75.00)$ & $3(25.00)$ & 12 & \\
\hline \multicolumn{5}{|c|}{ Lymph node metastasis } \\
\hline Yes & $38(90.48)$ & $4(9.52)$ & 42 & 0.001 \\
\hline No & $33(62.26)$ & $20(37.74)$ & 53 & \\
\hline \multicolumn{5}{|c|}{ Tumour size (diameter) } \\
\hline$<5 \mathrm{~cm}$ & $38(76.00)$ & $12(24.00)$ & 50 & 0.765 \\
\hline$\geq 5 \mathrm{~cm}$ & $33(73.33)$ & $12(26.67)$ & 45 & \\
\hline \multicolumn{5}{|c|}{ Serosa invasion (invasion depth) } \\
\hline Yes & $56(81.16)$ & $13(18.84)$ & 69 & 0.019 \\
\hline No & $15(57.69)$ & $11(42.31)$ & 26 & \\
\hline \multicolumn{5}{|l|}{ TNM stage } \\
\hline I-II & $32(64.00)$ & $18(36.00)$ & 50 & 0.011 \\
\hline III-IV & $39(86.67)$ & $6(13.33)$ & 45 & \\
\hline
\end{tabular}

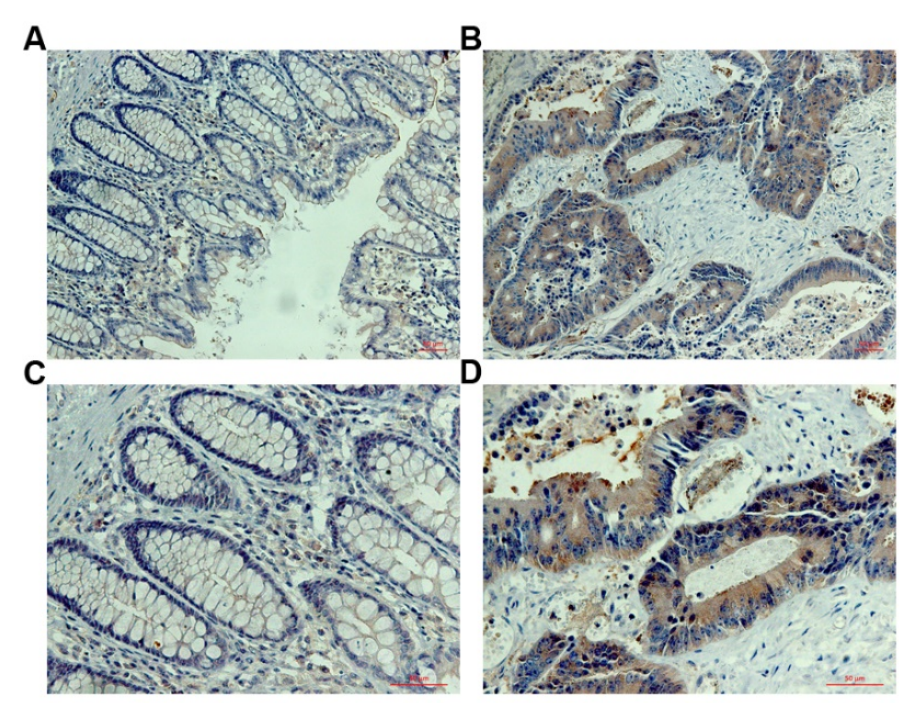

Figure 1. Immunohistochemistry assay 
A

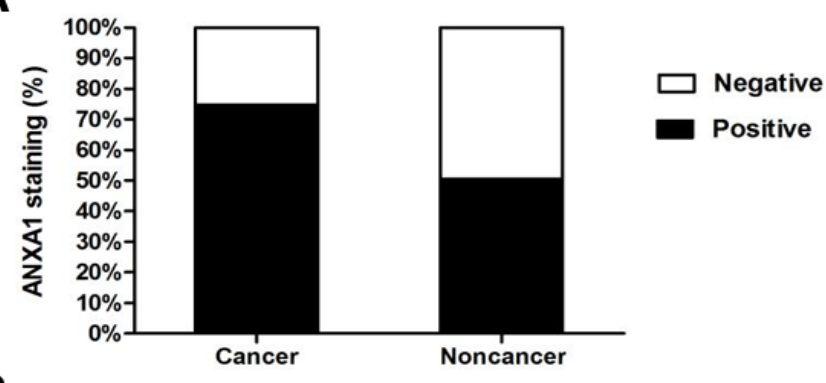

B

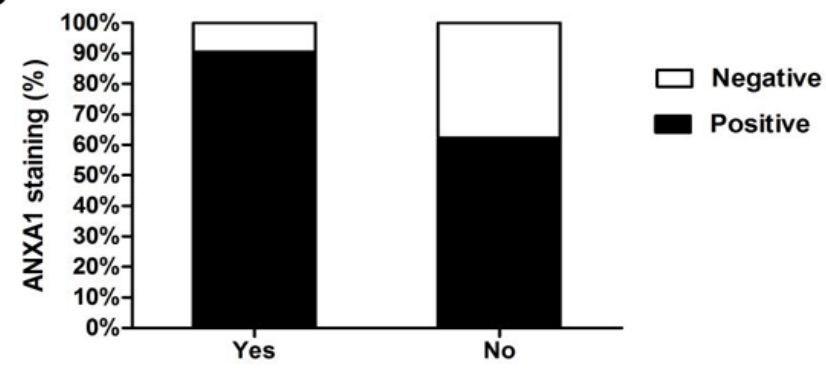

C

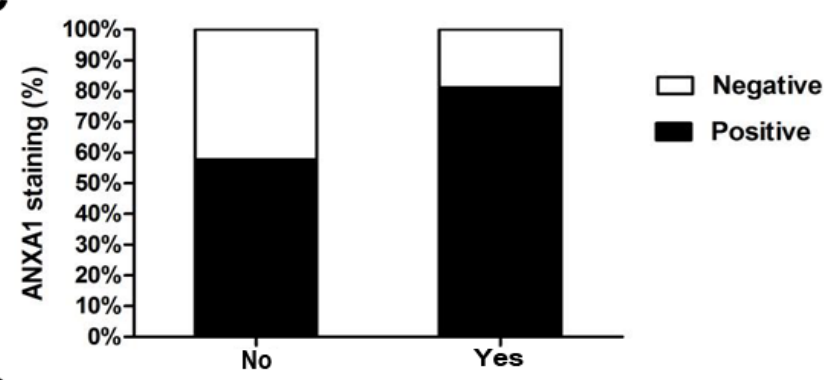

D

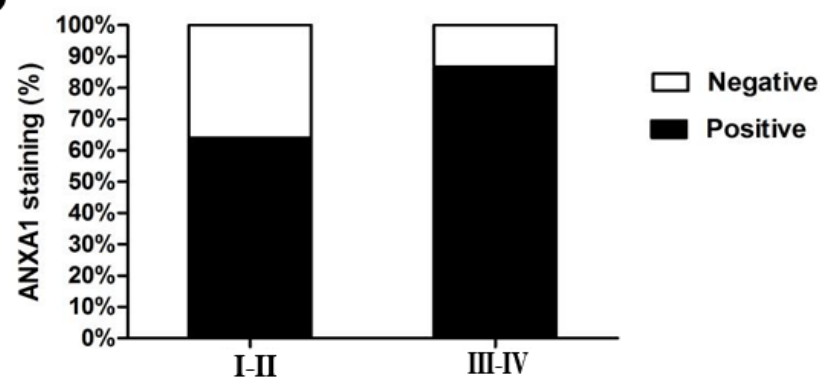

Figure 2. Increase in ANXA1 in CRC compared to non-cancerous tissues

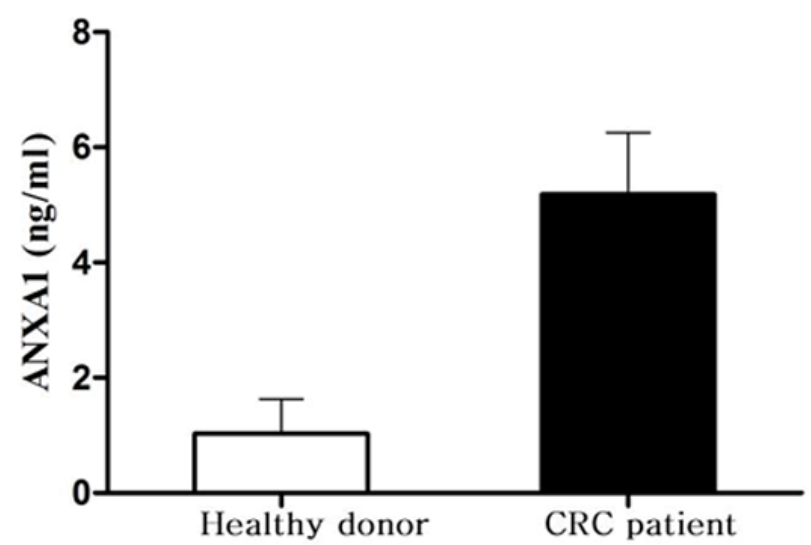

Figure 3. ANXA1 in peripheral blood of CRC patients and in healthy individuals by immunohistochemistry. In addition, the expression level of ANXA1 in peripheral blood of CRC patients was detected by ELISA. Our results showed that ANXA1 was increased in CRC tissues and upregulated in the peripheral blood of CRC patients. We provided evidences that ANXA1 upregulated in CRC and it may promote the development and growth of CRC.

Depth of tumor invasion has been assessed as an important prognostic marker for tumor progression and prognosis $[12,13]$. Tumor depth is a well-known prognostic factor in gastric cancer patients and was demonstrated that deeper tumor invasion was significantly associated with poor prognosis [13]. In CRC, pT1+pT2+pT3 $\leq 5 \mathrm{~mm}$ were subdivided in low risk group and $\mathrm{pT} 3>5 \mathrm{~mm}+\mathrm{pT} 4$ were subdivided in high risk group. Furthermore, the sensitivity and specificity of the two groups were 0.90 and 0.75 [14]. Our results demonstrated ANXA1 upregulation in CRC was related to deeper tumour invasion depth which suggested that ANXA1 may promote the invasion of CRC. Many experiments have demonstrated that tumor invasion depth and tumor size correlate closely with positive lymph node metastasis $[15,16]$.

Invasion and metastasis are the main causes of death for cancer patients and lymph node are the common sites of cancer metastasis [17]. Meanwhile, lymphatic metastasis predicts increased mortality of many cancer types. Although, the role of ANXA1 in tumor progress and metastasis also had inconsistent results $[9,18]$, there were more consistently results showed that upregulated ANXA1 related to cancer metastasis. Higher ANXA1 expressions were showed in lung metastases cell line compared with cell line from primary tumour and often higher stained in the lymph node metastases [10]. Our results demonstrated ANXA1 upregulation in CRC was related to lymph node metastasis. Our data suggested that ANXA1 may promote the metastases of CRC.

Increased migratory ability is one key step for tumour metastasis. Overexpression of ANXA1 could enhance cell migration of breast cancer cell line MCF-7 through ERK-RhoA-NFkB activation loop [19]. ANXA1 also could regulate mTOR signalling and the activity of MMP9 for regulating the migration activity of breast cancer cells, but which was not been uniformly supported [20]. EMT (epithelial mesenchymal transition), had been recognised playing vital role in tumour metastasis and there were evidences of relationships between ANXA1 and EMT in different subtypes of breast cancers [21-23]. The mechanism of ANXA1 regulating metastasis of CRC need further explored.

In most tumours, lymphatic metastasis plays an important role in tumour diffusion. The TNM (TNM Classification of Malignant Tumors) system underlines the importance of the depth of tumor invasion, lymphatic metastasis assessment and distant metastasis both in pathologic and in clinical setting [24]. The prognostic significance of lymphatic nodal involvement and TNM stage have been confirmed by many experiments that the outcome of tumor patients strongly depends on lymphatic nodal involvement and the stage of tumor at the time of diagnosis [25-27]. Our data also demonstrated ANXA1 upregulated in CRC was related to late TNM stage. Our data suggested that ANXA1 might have prognostic significance in CRC.

In summary, ANXA1 was upregulated in CRC tissues, and ANXA1 was correlated with deeper tumor invasion and lymph node metastasis of CRC. Furthermore, ANXA1 was correlated with late TNM stage of CRC patients. These results suggested that ANXA1 may promote the progression of CRC. In addition, high ANXA1 in peripheral blood may be considered as a biomarker for diagnosis of CRC. Our findings would provide new insights into significant of ANXA1 in diagnosis and prognosis of CRC. The underlying mechanism of ANXA1in CRC progression will be further explored. 


\section{Conflict of interest}

Qing-Hua Liu declares that she has no conflict of interest. Mei Ding declares that she has no conflict of interest. Jin-Min Sun declares that she has no conflict of interest. Zu-An Zhu declares that he has no conflict of interest.

\section{Acknowledgements}

This study was funded by grants from the National Natural Science Foundation of China (No. 81502030).

\section{References}

1. Arnold M, Sierra MS, Laversanne M, Soerjomataram I, Jemal A, et al. (2017) Global patterns and trends in colorectal cancer incidence and mortality. Gut 66: 683-691.

2. Watanabe T, Itabashi M, Shimada Y, Tanaka S, Ito Y, et al. (2015) Japanese Society for Cancer of the Colon and Rectum (JSCCR) Guidelines 2014 for treatment of colorectal cancer. Int J Clin Oncol 20: 207-239. [Crossref]

3. Lim LH, Pervaiz S (2007) Annexin 1: the new face of an old molecule. FASEB Journal 21: 968-975.

4. Garcia Pedrero JM, Fernandez MP, Morgan RO, Herrero Zapatero A, Gonzalez MV, et al. (2004) Annexin A1 down-regulation in head and neck cancer is associated with epithelial differentiation status. Am J Pathol 164: 73-79. [Crossref]

5. Hu N, Flaig MJ, Su H, Shou JZ, Roth MJ, et al. (2004) Comprehensive characterization of annexin I alterations in esophageal squamous cell carcinoma. Clinical cancer research 10: 6013-6022.

6. Biaoxue R, Xiguang C, Shuanying Y (2014) Annexin A1 in malignant tumors: current opinions and controversies. The International Journal of Biological Markers 29: e8-e20.

7. Guo C, Liu S, Sun MZ (2013) Potential role of Anxa1 in cancer. Future oncology 9:1773-1793.

8. Boudhraa Z, Bouchon B, Viallard C, D'Incan M, Degoul F (2016) Annexin A1 localization and its relevance to cancer. Clinical science 130: 205-220.

9. Ahn SH, Sawada H, Ro JY, Nicolson GL (1997) Differential expression of annexin I in human mammary ductal epithelial cells in normal and benign and malignant breast tissues. Clinical \& Experimental Metastasis 15: 151-156.

10. Pencil SD, Toth M (1998) Elevated levels of annexin I protein in vitro and in vivo in rat and human mammary adenocarcinoma. Clinical \& Experimental Metastasis 16: 113-121.

11. Ang EZ, Nguyen HT, Sim HL, Putti TC, Lim LH (2009) Annexin-1 regulates growth arrest induced by high levels of estrogen in MCF-7 breast cancer cells. Molecular Cancer Research 7: 266-274.

12. Ohashi T, Komatsu S, Ichikawa D, Kosuga T, Okamoto K, et al. (2016) Tumor Index as a Combined Indicator of Tumor Depth and Size in Gastric Cancer. Anticancer research 36: $1895-1900$.
13. Haraguchi N, Arigami T, Uenosono Y, Yanagita S, Uchikado Y, et al. (2018) Clinical significance of primary tumor score determined by tumor depth and size in patients with resectable gastric cancer. Oncotarget 9: 8512-8520.

14. Malmstrom ML, Gogenur I, Riis LB, Hassan H, Klausen TW, et al. (2017) Endoscopic ultrasonography and computed tomography scanning for preoperative staging of colonic cancer. International Journal of Colorectal Disease 32: 813-820.

15. Huang CM, Xu M, Wang JB, Zheng CH, Li P, et al. (2014) Is tumor size a predictor of preoperative N staging in T2-T4a stage advanced gastric cancer? Surgical Oncology 23: $5-10$.

16. Beaton C, Twine CP, Williams GL, Radcliffe AG (2013) Systematic review and metaanalysis of histopathological factors influencing the risk of lymph node metastasis in early colorectal cancer. Colorectal Disease 15: 788-797.

17. Chaffer CL, Weinberg RA (2011) A perspective on cancer cell metastasis. Science 331: 1559-1564. [Crossref]

18. Shen D, Nooraie F, Elshimali Y, Lonsberry V, He J, et al. (2006) Decreased expression of annexin A1 is correlated with breast cancer development and progression as determined by a tissue microarray analysis. Human Pathology 37: 1583-1591.

19. Bist P, Phua QH, Shu S, Yi Y, Anbalagan D, et al. (2015) Annexin-A1 controls an ERK-RhoA-NFkappaB activation loop in breast cancer cells. Biochemical and Biophysical Research Communications 461: 47-53.

20. Bhardwaj A, Ganesan N, Tachibana K, Rajapakshe K, Albarracin CT, et al. (2015) Annexin A1 Preferentially Predicts Poor Prognosis of Basal-Like Breast Cancer Patients by Activating mTOR-S6 Signaling. PloS One 10: e0127678. [Crossref]

21. Okano M, Kumamoto K, Saito M, Onozawa H, Saito K, et al. (2015) Upregulated Annexin A1 promotes cellular invasion in triple-negative breast cancer. Oncology Reports 33: 1064-1070.

22. Maschler S, Gebeshuber CA, Wiedemann EM, Alacakaptan M, Schreiber M, et al (2010) Annexin A1 attenuates EMT and metastatic potential in breast cancer. EMBO Molecular Medicine 2: 401-414.

23. de Graauw M, van Miltenburg MH, Schmidt MK, Pont C, Lalai R, et al. (2010) Annexin A1 regulates TGF-beta signaling and promotes metastasis formation of basallike breast cancer cells. Proc Natl Acad Sci USA 107: 6340-6345. [Crossref]

24. Ruffini E, Filosso PL, Guerrera F, Lausi P, Lyberis P, et al. (2018) Optimal surgical approach to thymic malignacies: New trends challenging old dogmas. Lung cancer 118: $161-170$

25. Weksler B, Holden A, Sullivan JL (2015) Impact of Positive Nodal Metastases in Patients with Thymic Carcinoma and Thymic Neuroendocrine Tumors. J Thorac Oncol 10: 1642-1647. [Crossref]

26. Weksler B, Pennathur A, Sullivan JL, Nason KS (2015) Resection of thymoma should include nodal sampling. The Journal of Thoracic and Cardiovascular Surgery 149: $737-742$

27. Hwang Y, Park IK, Park S, Kim ER, Kang CH, et al. (2016) Lymph Node Dissection in Thymic Malignancies: Implication of the ITMIG Lymph Node Map, TNM Stage Classification, and Recommendations. Journal of Thoracic Oncology 11: 108-114.

Copyright: $₫ 2018$ Qing-Hua L. This is an open-access article distributed under the terms of the Creative Commons Attribution License, which permits unrestricted use, distribution, and reproduction in any medium, provided the original author and source are credited. 